\title{
Waist-to-Height Ratio as a Simple and Useful Indicator for Non-alcoholic Fatty Liver Disease in Korean Women
}

\author{
Hyun-Jae Lim, Min-Seok Seo, Hye-Ree Lee, Jae-Yong Shim, Hee-Taik Kang, Yong-Jae Lee* \\ Department of Family Medicine, Gangnam Severance Hospital, Yonsei University College of Medicine, Seoul, Korea
}

Background: It has not been determined which obesity index might be most appropriate to predict nonalcoholic fatty liver disease in Asian populations. This study aimed to evaluate the usefulness of the waist-to-height ratio in assessing patients with nonalcoholic fatty liver disease and to identify the optimal cut-off values useful for predicting nonalcoholic fatty liver disease.

Methods: Receiver operating characteristic curve analyses were conducted in order to assess the accuracy of the waist circumference, body mass index, and waist-to-height ratio for detecting nonalcoholic fatty liver disease among 616 women aged 20 years or older. To evaluate the optimal value of anthropometric indices, the Youden J-index (sensitivity+specificity-1) was used.

Results: The area under the ROC curve of waist-to-height ratio was highest among anthropometric obesity indices as follows: 0.776 (0.731$0.822)$ for waist circumference, $0.775(0.728-0.822)$ for body mass index, and 0.792 (0.748-0.836) for waist-to-height ratio, respectively. Using a waist-to-height ration cut-off value of 0.49 , the sensitivity and specificity for detecting nonalcoholic fatty liver disease were $72.3 \%$ and $74.7 \%$, respectively.

Conclusion: These results demonstrated that the waist-to-height ratio may be a better obesity index for identifying individuals at risk for nonalcoholic fatty liver disease in Korean women.

Key words: Anthropometry, Obesity, Waist-to-height ratio, Nonalcoholic fatty liver disease

\section{Introduction}

Non-alcoholic fatty liver disease (NAFLD) is the most common clinicopathologic form of chronic liver disease. NAFLD is characterized by significant lipid accumulation in hepatocytes and persistent elevation in liver enzymes without excessive alcohol intake, or other common causes of liver disease. The increasing prevalence of NAFLD is a serious and growing worldwide public health problem because of its potential to progress to end-stage liver disease, such as advanced cirrhosis, hepatic failure, and hepatocellular carcinoma. ${ }^{1,2}$ This topic has been extensively studied, and research has shown that NAFLD also predicts an increased risk of developing type 2 diabetes, mellitus, metabolic syndrome, and cardiovascular disease (CVD), which is a leading cause of death among both adults and children, particularly in developed countries. ${ }^{3,4}$ Thus, early identification of NAFLD is important in order to prevent further progression.

The previous literature has demonstrated that obesity is clearly linked to NAFLD, and visceral fat is more directly related to the pathogenesis of NAFLD. ${ }^{5}$ Abdominal obesity has been recognized as a better predictor for CVD and metabolic morbidities, as compared to the body mass index (BMI). Waist circumference (WC) has become a widely used measurement for quantifying abdominal fat accumulation. However, ethnicity and sex-specific cut-off thresholds for WC should be taken into account when considering the prediction of cardiometabolic diseases such as metabolic syndrome. Recently, the waist-to-height ratio (WHtR) has been suggested as an alternative anthropometric index for central adiposity. Epidemiological studies reported that WHtR appears to be more strongly associ-
Corresponding author Yong-Jae Lee iD http://orcid.org/0000-0002-6697-476X Department of Family Medicine, Yonsei University College of Medicine, 211 Eonju-ro, Gangnam-gu, Seoul 06273, Korea

Tel +82-2-2019-3480 Fax +82-2-3463-3287 E-mail ukyjhome@yuhs.ac Hyun-Jae Lim and Min-Seok Seo contributed equally to this work. Received Apr. 23, 2015 Reviewed Jun. 24, 2015 Accepted Aug. 17, 2015
Copyright (C) 2016 Korean Society for the Study of Obesity

(c) This is an Open Access article distributed under the terms of the Creative Commons Attribution Non-Commercial License (http://creativecommons.org/licenses/by-nc/3.0/) which permits unrestricted non-commercial use, distribution, and reproduction in any medium, provided the original work is properly cited. 
ated with obesity-related diseases and metabolic risk factors than other obesity indices. ${ }^{6,7}$ However, there is little evidence regarding the accuracy of using the WHtR as a predictor for NAFLD. This study evaluated the accuracy of the WHtR as a predictor for NAFLD and attempted to identify the optimal cut-off values for predicting NAFLD in Korean women.

\section{Methods}

\section{Study participants}

Nine hundred sixty participants over the age of 20 underwent medical examinations at the Health Promotion Center of Gangnam Severance Hospital located in Seoul, Korea, from March 2010 to May 2011. Participants with missing covariate data $(n=56)$, alcohol consumption of more than $70 \mathrm{~g} /$ week $(\mathrm{n}=91)$, and positive serologic results for either the hepatitis B antigen or the hepatitis C antibody were excluded from this study, as well as participants who had a history of chronic liver disease $(\mathrm{n}=56)$. Additionally, subjects with a history of cancer, CVD, cerebrovascular, respiratory, or rheumatologic disease $(n=97)$ were excluded. As a result, 616 participants were included in the final analysis. This study was approved by the Institutional Review Board of Yonsei University College of Medicine located in Seoul, Korea.

\section{Data collection}

All participants completed self-reported questionnaires about their lifestyle (alcohol consumption, cigarette smoking, and physical activity) and medical history. Anthropometric data (body weight and height) were measured in light clothing without shoes to the nearest $0.1 \mathrm{~kg}$ and $0.1 \mathrm{~cm}$, respectively. The WC was measured at the midpoint between the lower margin of the rib cage and the iliac crest in a standing position. The BMI was calculated as the weight in kilograms divided by the square of the height in meters $\left(\mathrm{kg} / \mathrm{m}^{2}\right)$. The WHtR was calculated by dividing the WC $(\mathrm{cm})$ by the height $(\mathrm{cm})$. Systolic blood pressure (SBP) and diastolic blood pressure (DBP) were measured in the right arm using a standard mercury sphygmomanometer (Baumanometer, Baum, Copiague, NY, USA). All blood samples were collected from the antecubital vein after fasting for at least 12 hours. Fasting blood glucose, total cholesterol, triglyceride, high-density lipoprotein (HDL)-cholesterol, aspartate aminotransferase (AST), and alanine aminotransferase (ALT) levels were analyzed by enzymatic methods using an automatic chemistry analyzer (Hitachi 7600-110, Tokyo, Japan).

A diagnosis of a fatty liver was based on abdominal ultrasonography with a 3.5-MHz transducer (HDI 5000, Philips, Bothell, USA); two experienced radiologists performed the ultrasonography. They were unaware of both the purposes of this study, as well as the laboratory findings. The coefficients of variation (CVs) for inter- and intraoperator reproducibility were $6.8 \%$ and $4.3 \%$, respectively. Hepatic steatosis was determined to be present when high hepatorenal echo contrast, bright liver parenchyma, or attenuation of ultrasound in a deep area of the liver was detected. Based on the criteria described previously, hepatic steatosis was graded into different categories as follows: (a) mild degree, which is characterized by a slight diffuse increase in bright fine echoes in the liver parenchyma, normal visualization of the diaphragm border and intrahepatic vessel borders, and normal hepatorenal contrast; (b) moderate degree, which is characterized by a diffuse increase in bright fine echoes in the liver parenchyma and slightly impaired visualization of the diaphragm border and intrahepatic vessel borders; or (c) severe degree, which is characterized by a marked increase in bright fine echoes at a shallow depth with deep attenuation, impaired visualization of the diaphragm and the back part of right liver lobe, and marked vascular blurring. ${ }^{8,9}$

\section{Statistical analysis}

Continuous variables were characterized by the mean and the standard error, and categorical variables were presented as an estimated proportion. The clinical characteristics of the study population that had NAFLD were analyzed using an independent twosample $t$-test for the continuous variables and a chi-square test for the categorical variables. Receiver operating characteristic (ROC) curve analyses were generated to evaluate the optimal cutoff points of the WC, BMI, and WHtR for detecting participants who had NAFLD. To evaluate the optimal value of the anthropometric indices, the Youden J-index (sensitivity+specificity-1) was used. The area under the ROC curve (AUROC) was used to compare the diagnostic powers of the anthropometric indices. All analyses were conducted using the SAS statistical software (version 9.2, SAS Institute Inc., Cary, NC, USA) and the STATA statistical software (version 13.0, STATA Corporation, TX, USA). A two-sided test was performed and statistical significance was determined at a $P$ value of less than 0.05 . 
Table 1. Characteristics of the study participants according to the diagnosis of NAFLD

\begin{tabular}{lccc}
\hline & NAFLD (-) & NAFLD (+) & $P$ value* \\
\hline $\mathrm{N}$ & 506 & 110 & NA \\
Age (yr) & $45.0 \pm 8.0$ & $48.4 \pm 7.5$ & $<0.01$ \\
Current smoker (\%) & 5.3 & 0.9 & 0.13 \\
Regular exercise (\%) & 49.4 & 33.6 & $<0.01$ \\
Height (cm) & $158.9 \pm 5.0$ & $157.1 \pm 5.2$ & $<0.01$ \\
Body weight (kg) & $57.7 \pm 7.1$ & $64.0 \pm 7.9$ & $<0.01$ \\
Waist circumference (cm) & $73.9 \pm 7.1$ & $81.9 \pm 7.8$ & $<0.01$ \\
Body mass index (kg/m²) & $22.8 \pm 2.6$ & $25.9 \pm 3.3$ & $<0.01$ \\
Waist-to-height ratio & $0.47 \pm 0.05$ & $0.52 \pm 0.05$ & $<0.01$ \\
Systolic blood pressure (mmHg) & $116.6 \pm 15.3$ & $125.1 \pm 14.2$ & $<0.01$ \\
Diastolic blood pressure (mmHg) & $72.0 \pm 9.4$ & $77.8 \pm 9.1$ & $<0.01$ \\
Fasting plasma glucose (mg/dL) & $86.6 \pm 13.9$ & $90.8 \pm 9.6$ & $<0.01$ \\
Total cholesterol (mg/dL) & $177.4 \pm 30.1$ & $200.3 \pm 38.0$ & $<0.01$ \\
Triglyceride (mg/dL) & $93.7 \pm 46.5$ & $148.9 \pm 79.6$ & $<0.01$ \\
HDL cholesterol (mg/dL) & $59.1 \pm 12.1$ & $51.1 \pm 9.7$ & $<0.01$ \\
LDL cholesterol (mg/dL) & $102.6 \pm 27.9$ & $124.0 \pm 39.9$ & $<0.01$ \\
AST (IU/L) & $18.4 \pm 6.1$ & $21.4 \pm 8.6$ & $<0.01$ \\
ALT (IU/L) & $15.7 \pm 9.3$ & $23.8 \pm 15.9$ & $<0.01$ \\
\hline
\end{tabular}

NA, not applicable; HDL, high density lipoprotein; LDL, low density lipoprotein; AST, aspartate aminotransferase; ALT, alanine aminotransferase.

${ }^{*} P$ values were calculated by an independent two-sample $t$-test or chi-square test.

\section{Results}

The clinical characteristics of the study participants that had NAFLD are shown in Table 1 . The overall prevalence of NAFLD was observed to be $17.9 \%$. Subjects with NAFLD had higher anthropometric indices, including WC, BMI and WHtR. In addition, blood pressure, fasting plasma glucose, total cholesterol, triglyceride, AST, and ALT were higher in the NAFLD group.

Fig. 1 shows the ROC curves used to evaluate the accuracy of obesity indices, in order to identify participants with NAFLD. The AUROC of the WHtR was highest among anthropometric obesity indices as follows: 0.776 (0.731-0.822) for WC, 0.775 (0.728-0.822) for BMI, and 0.792 (0.748-0.836) for WHtR, respectively.

The optimal cut-off values of the WHtR, BMI and WC for detecting NAFLD were $76.5 \mathrm{~cm}$ for WC, $23.0 \mathrm{~kg} / \mathrm{m}^{2}$ for BMI, and 0.49 for WHtR. The highest sensitivity and specificity for detecting NAFLD were $72.3 \%$ and $74.7 \%$, respectively, using a WHtR cutoff value of 0.49 (Table 2).

\section{Discussion}

Given the growing prevalence and clinical implications of

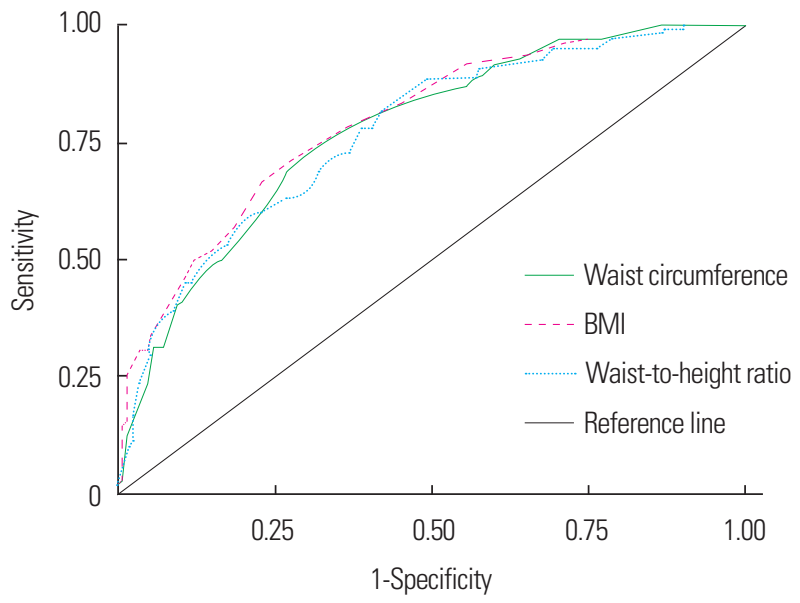

Fig. 1. The receiver operating characteristic $(\mathrm{ROC})$ curves for the waist-to-height ratio, body mass index, and waist circumference to predict non-alcoholic fatty liver disease.

Table 2. Area under the ROC curve (AUROC) and optimal cut-off points for waist circumference, waist-to-height ratio, and BMI to detect NAFLD

\begin{tabular}{lccccc}
\hline & AUROC (95\% Cl) & Cut-off point & Sensitivity & Specificity & $\begin{array}{c}\text { Youden } \\
\text { J-index* }\end{array}$ \\
\hline WC (cm) & $0.776(0.731-0.822)$ & 76.5 & 76 & 67 & 0.43 \\
BMI (kg/m²) & $0.775(0.728-0.822)$ & 23.0 & 85 & 56 & 0.41 \\
WHtR & $0.792(0.748-0.836)$ & 0.49 & 72 & 74 & 0.46 \\
\hline
\end{tabular}

WC, waist circumference; BMI, body mass index; WHtR, waist-to-height ratio.

*Youden J-index=Sensitivity+Specificity-1.

NAFLD, the use of a simple and useful tool for the early identification of NAFLD is beneficial from a public health perspective. Among the three anthropometric indices studied here, the WHtR had the largest AUROC, with a cut-off value of 0.49 used for detecting NAFLD in Korean women. Our results are in agreement with the results of previous studies conducted in Japan, China and other countries. Several previous studies have shown that a WHtR close to 0.5 has a stronger relationship with cardiovascular and metabolic diseases. Hsieh et al. ${ }^{10}$ used ROC curve analysis in Japanese men and women to determine a WHtR cut-off value of 0.5 to indicate a clustering of CVD risk factors. Moreover, Ko et al. ${ }^{11}$ proposed WHtR cut-off values of 0.48-0.51 in men and 0.49-0.52 in women in order to predict hypertension, type 2 diabetes, dyslipidemia, and albuminuria in a Chinese population study. Ashwell et al. ${ }^{12}$ has also suggested the same WHtR of 0.5 as a boundary value of CVD risk in a representative sample of British adults aged 19-64 years.

Several mechanisms could explain the relationship between central obesity and NAFLD. Visceral adipocytes may be directly related to NAFLD due to their capability to synthesize, modulate, and se- 
crete cytokines and adipocytokines. Macrophages accumulate in the adipocytes of obese patients, and this process is accelerated in visceral fat. These macrophages are likely to produce pro-inflammatory cytokines and several adipocytokines, such as leptin, resistin, and tumor necrosis factor alpha (TNF- $\alpha){ }^{13,14}$ Disturbed adipocytokine secretion is involved in the regulation of insulin resistance and may promote hepatic steatosis in the key pathophysiology of NAFLD.

Generally, the BMI has been the most commonly used anthropometric parameter to reflect body fat mass. However, the BMI is limited because it does not take into account the how the body fat is distributed, i.e., it does not consider various occurrences such as central adiposity. ${ }^{15,16}$ Therefore, it is insufficient to determine the obesity-related disease risk using the BMI alone; other anthropometric parameters are necessary to evaluate this risk. In this regard, WC has been recommended to be a good indicator of central obesity. However, WC does not account for differences in height. The height is an important parameter that should be considered before adopting an obesity index because height may reflect different body fat distributions, in addition to overall adiposity. When sex and ethnicity specific WC is used as an obesity indicator, individuals of relatively taller stature may have an overestimated obesity-related disease risk, while short-statured individuals with the same WC may have an underestimated risk. ${ }^{17}$ In this regard, the WHtR and the WC adjusted for height may be alternative, practical indices useful for the screening of abdominal obesity, due to how these measures allow for individuals with different heights to have their own cut-off WCs, particularly in populations with a wide range of ages and heights. Moreover, the diagnostic strength of WHtR includes closer cut-off points between men and women than does WC, so a single cut-off value near 0.5 may be applied independently of age, sex, and ethnicity.

The present study had some limitations to be considered. First, as this study was a cross-sectional study, it had a limited ability to explain a causal relationship between anthropometric indices and the risk of NAFLD. Second, the study subjects were volunteers visiting for a promotional health screening at a single hospital. It is possible that these individuals were slightly healthier than the general public. As a result of this potential selection bias, the study findings may not be as applicable to the general population in Korea. Third, we did not perform liver biopsies for the diagnosis of fatty liver, even though liver biopsy is considered the gold standard for the diagnosis of NAFLD. We chose not to use liver biopsies as liver biopsies are an invasive pro- cedure in the standard clinical setting, and ultrasonography is a noninvasive yet widely available method used for the qualitative assessments of hepatic fat accumulation. Thus, ultrasonography is the preferred modality for mass screening for hepatic steatosis with a reasonable accuracy (67-94\%). ${ }^{8,18}$

In summary, a WHtR cut-off point near 0.5 has the potential to be a simple and practical index to identify NAFLD in Korean women.

\section{Conflicts of Interest}

The authors declared no potential conflicts of interests of interesting with respect to the research, authorship, and/or publication of this article.

\section{Acknowledgments}

This study was supported by a new faculty research seed money grant of Yonsei University College of Medicine for 2015 (2015-320009).

\section{References}

1. Angulo P. GI epidemiology: nonalcoholic fatty liver disease. Aliment Pharmacol Ther 2007;25:883-9.

2. Matteoni CA, Younossi ZM, Gramlich T, Boparai N, Liu YC, McCullough AJ. Nonalcoholic fatty liver disease: a spectrum of clinical and pathological severity. Gastroenterology 1999;116:1413-9.

3. Marchesini G, Brizi M, Bianchi G, Tomassetti S, Bugianesi E, Lenzi M, et al. Nonalcoholic fatty liver disease: a feature of the metabolic syndrome. Diabetes 2001;50:1844-50.

4. Targher G, Day CP, Bonora E. Risk of cardiovascular disease in patients with nonalcoholic fatty liver disease. N Engl J Med 2010; 363:1341-50.

5. van der Poorten D, Milner KL, Hui J, Hodge A, Trenell MI, Kench JG, et al. Visceral fat: a key mediator of steatohepatitis in metabolic liver disease. Hepatology 2008;48:449-57.

6. Ashwell M, Gunn P, Gibson S. Waist-to-height ratio is a better screening tool than waist circumference and BMI for adult cardiometabolic risk factors: systematic review and meta-analysis. Obes Rev 2012;13:275-86.

7. Hsieh SD, Yoshinaga H, Muto T. Waist-to-height ratio, a simple 
and practical index for assessing central fat distribution and metabolic risk in Japanese men and women. Int J Obes Relat Metab Disord 2003;27:610-6.

8. Saverymuttu SH, Joseph AE, Maxwell JD. Ultrasound scanning in the detection of hepatic fibrosis and steatosis. Br Med J (Clin Res Ed) $1986 ; 292: 13-5$.

9. Saadeh S, Younossi ZM, Remer EM, Gramlich T, Ong JP, Hurley M, et al. The utility of radiological imaging in nonalcoholic fatty liver disease. Gastroenterology 2002;123:745-50.

10. Hsieh SD, Yoshinaga $\mathrm{H}$. Abdominal fat distribution and coronary heart disease risk factors in men-waist/height ratio as a simple and useful predictor. Int J Obes Relat Metab Disord 1995;19:585-9.

11. Ko GT, Chan JC, Cockram CS, Woo J. Prediction of hypertension, diabetes, dyslipidaemia or albuminuria using simple anthropometric indexes in Hong Kong Chinese. Int J Obes Relat Metab Disord 1999;23:1136-42.

12. Ashwell M, Gibson S. Waist to height ratio is a simple and effective obesity screening tool for cardiovascular risk factors: analysis of data from the British National Diet and Nutrition Survey of adults aged 19-64 years. Obes Facts 2009;2:97-103.

13. Tilg H. Adipocytokines in nonalcoholic fatty liver disease: key players regulating steatosis, inflammation and fibrosis. Curr Pharm Des 2010;16:1893-5.

14. Kumar R, Prakash S, Chhabra S, Singla V, Madan K, Gupta SD, et al. Association of pro-inflammatory cytokines, adipokines \& oxidative stress with insulin resistance \& non-alcoholic fatty liver disease. Indian J Med Res 2012;136:229-36.

15. Dalton M, Cameron AJ, Zimmet PZ, Shaw JE, Jolley D, Dunstan DW, et al. Waist circumference, waist-hip ratio and body mass index and their correlation with cardiovascular disease risk factors in Australian adults. J Intern Med 2003;254:555-63.

16. Deurenberg-Yap M, Chew SK, Deurenberg P. Elevated body fat percentage and cardiovascular risks at low body mass index levels among Singaporean Chinese, Malays and Indians. Obes Rev 2002; 3:209-15.

17. Misra A, Wasir JS, Vikram NK. Waist circumference criteria for the diagnosis of abdominal obesity are not applicable uniformly to all populations and ethnic groups. Nutrition 2005;21:969-76.

18. Graif M, Yanuka M, Baraz M, Blank A, Moshkovitz M, Kessler A, et al. Quantitative estimation of attenuation in ultrasound video images: correlation with histology in diffuse liver disease. Invest Radiol 2000;35:319-24. 